\title{
Optimal Quantum Spatial Search with One-Dimensional Long-Range Interactions
}

\author{
Dylan Lewis $\odot,{ }^{1, *}$ Asmae Benhemou $\odot,{ }^{1}$ Natasha Feinstein $\odot,{ }^{1}$ Leonardo Banchi $\odot,{ }^{2,3}$ and Sougato Bose ${ }^{1}$ \\ ${ }^{1}$ Department of Physics and Astronomy, University College London, London WCIE 6BT, United Kingdom \\ ${ }^{2}$ Department of Physics and Astronomy, University of Florence, via G. Sansone 1, I-50019 Sesto Fiorentino (Florence), Italy \\ ${ }^{3}$ INFN Sezione di Firenze, via G. Sansone 1, I-50019 Sesto Fiorentino (Florence), Italy
}

(Received 29 October 2020; revised 9 April 2021; accepted 7 May 2021; published 17 June 2021)

\begin{abstract}
Continuous-time quantum walks can be used to solve the spatial search problem, which is an essential component for many quantum algorithms that run quadratically faster than their classical counterpart, in $\mathcal{O}(\sqrt{n})$ time for $n$ entries. However, the capability of models found in nature is largely unexplored-e.g., in one dimension only nearest-neighbor Hamiltonians have been considered so far, for which the quadratic speedup does not exist. Here, we prove that optimal spatial search, namely with $\mathcal{O}(\sqrt{n})$ run time and high fidelity, is possible in one-dimensional spin chains with long-range interactions that decay as $1 / r^{\alpha}$ with distance $r$. In particular, near unit fidelity is achieved for $\alpha \approx 1$ and, in the limit $n \rightarrow \infty$, we find a continuous transition from a region where optimal spatial search does exist $(\alpha<1.5)$ to where it does not $(\alpha>1.5)$. Numerically, we show that spatial search is robust to dephasing noise and that, for reasonable chain lengths, $\alpha \lesssim 1.2$ should be sufficient to demonstrate optimal spatial search experimentally with near unit fidelity.
\end{abstract}

DOI: 10.1103/PhysRevLett.126.240502

Introduction.-Spatial search is the problem of finding a marked element in a graph with $n$ nodes. For classical algorithms, there is no shortcut and $\mathcal{O}(n)$ queries are required. However, with quantum algorithms, spatial search can be solved optimally in $\mathcal{O}(\sqrt{n})$ time [1,2]. Childs and Goldstone [3] found that spatial search can be solved by an algorithm using a continuous-time quantum walk. They showed that for the complete graph, the hypercube graph, and $d$-dimensional periodic lattices of $d>4$, the marked node can be found in optimal time. Since then, a number of graphs have been found that permit optimal spatial search [4-10]. Recently, Chakraborty et al. [11] have shown necessary and sufficient conditions for optimal spatial search for graphs with a sufficiently large spectral gap.

A quantum walk is the quantum equivalent of the classical random walk. Unlike a classical random walk, the quantum walker takes a superposition of paths [12]. The interference between those paths forms the basis of the quantum algorithms that utilize quantum walks. From a graph-theoretical perspective, a continuous-time quantum walk is generated by a unitary evolution defined by the adjacency matrix $A$ of a graph $[13,14]$. The vertices of the graph define orthonormal basis states of a Hilbert space and an evolution for time $t$ is given by $e^{-i A t}$. The latter is equivalent to the natural dynamics of a quantum system where the Hamiltonian is the adjacency matrix of the graph that defines the hopping between basis states.

Can Hamiltonians found in nature, with interactions typically falling off with distance, admit a quantum walk capable of optimal spatial search? This is an important question in the noisy intermediate-scale quantum (NISQ)
[15] era where we can look for the quantum speedup capabilities of non-error-corrected collections of qubits with physically motivated couplings. Most of the previously studied Hamiltonians that admit optimal spatial search are difficult to find in nature and must be artificially synthesized, e.g., using quantum simulation techniques [16] or a digital quantum computer. Even a recent idea of using an unweighted long-range percolation graph [17] would amount to having stochastic interactions, whose realization is unclear. Childs and Ge [18] also noted that an interaction strength that decays as a quadratic power law with distance would be sufficient to find optimal spatial search in $d=2$. For one-dimensional systems, only nearest-neighbor interactions have been considered, where optimal quantum search was shown to be impossible [3].

Here, we propose a physically motivated model for spatial search on a closed one-dimensional spin chain using long-range interactions that decay as $1 / r^{\alpha}$, with $r=$ $|i-j|$ the distance between lattice sites $i$ and $j$, and $\alpha \geq 0$. At the moment, this is a highly topical model, realizable in ion traps [19-22], dipolar crystals [23,24], Rydberg arrays [25] etc., although the tunability of $\alpha$ is probably present only in the ion trap setting. This model has been explored for its capabilities of scrambling [26], novel dynamical quantum phase transitions [27], and quantum state transfer $[28,29]$. Yet its potential for quantum computation remains unexplored.

In the case $\alpha=0$, we have the complete graph. This is equivalent to nearest-neighbor interactions in a spatial dimension equal to the number of spins $n$. For complete graphs, optimal spatial search has been shown [30]. In the 
case of large $\alpha$, the graph approaches the one-dimensional periodic lattice, where spatial search does not exist. This picture, where long-range interactions effectively mask the dimension of the system [31], suggests there is a transition between the regime where optimal spatial search exists and where it does not. Here, we address the following questions: for which values of $\alpha$ can we show optimal search, and at what value of $\alpha$ is there a transition between the two regimes? We will show both numerically and analytically for large $n$ that optimal spatial search, namely with $\mathcal{O}(\sqrt{n})$ run time, does exist for $\alpha<1.5$ and has a near perfect fidelity for $\alpha \lesssim 1.2$. We note that the interaction strengths found for optimal search are experimentally realizable. In particular, ion trap experiments have been performed for chains of ions with interaction strengths with a potential of $\alpha \approx 1$ [19-22]. In principle, $\alpha$ can be tuned to anywhere between 0 and 3 for low $n$ [21]. However, as $n$ increases, $\alpha \ll 1$ becomes experimentally more difficult. We show that experimental designs using ion traps along these lines would be able to demonstrate optimal spatial search.

Spatial search.-A quantum search problem can be solved in $\mathcal{O}(\sqrt{n})$ time using Grover's algorithm [1]. An analog version of this search algorithm was suggested by Farhi and Gutmann [30], which is a continuous-time quantum walk on a complete graph [3]. It is experimentally difficult to encode this search in the general case for the entire Hilbert space, which gives a graph of size $2^{n}$ for $n$ spins, because it is hard to implement the continuous-time oracle Hamiltonian and the graph Hamiltonian. We therefore restrict ourselves to the spatial search problem in the single-excitation basis of size $n$, which can naturally be mapped to a physical setting. Each of the $n$ vertices of the search graph, $G$, represent a single excited spin. The basis states of this space are therefore $|j\rangle=|0\rangle_{1} \otimes \ldots \otimes$ $|0\rangle_{j-1} \otimes|1\rangle_{j} \otimes|0\rangle_{j+1} \otimes \ldots \otimes|0\rangle_{n}=|0 \ldots 010 \ldots 0\rangle$. The marked state is identified by measuring the system to locate the excited spin. The oracle Hamiltonian is simply a local magnetic field at the marked spin site,

$$
H_{\text {marked }}=|w\rangle\langle w|,
$$

where $w \in\{1, \ldots, n\}$ labels the marked vertex of the graph. The search includes the graph Hamiltonian and the marked state Hamiltonian,

$$
H_{\text {search }}=\gamma H+H_{\text {marked }},
$$

where the relative strength of the two Hamiltonians is given by $\gamma$, an effective hopping rate for the quantum walker between vertices of the graph. We use the original Childs and Goldstone spatial search algorithm for continuous-time quantum walks. First, a specific value of $\gamma$ is chosen. Then the system is initialized in a specific state $|s\rangle$ and evolved under the system dynamics for a time $T$. Finally, the state is measured and the marked state is found with probability $F$,

$$
F=\left|\left\langle w\left|e^{-i H_{\text {search }} T}\right| s\right\rangle\right|^{2} .
$$

The aim is to find an $F$ as close to 1 as possible. The search is optimal if $T$ is $\mathcal{O}(\sqrt{n})$. The initial state is

$$
|s\rangle=\frac{1}{\sqrt{n}} \sum_{j=1}^{n}|j\rangle,
$$

which has an overlap of $1 / \sqrt{n}$ with the marked state.

For spatial search, we want the system to oscillate between the states $|s\rangle$ and $|w\rangle$ with high fidelity $[32,33]$. The $\gamma$ that maximizes the overlap of the dominant eigenvectors of the system with the states $|s\rangle$ and $|w\rangle$ achieves this [3]. The maximum overlap occurs at the minimum gap, that is the minimum energy difference between the ground state energy and the first excited state energy. The time it takes to oscillate between the superposition state and the marked state is proportional to the inverse of the energy gap. The minimum energy gap is proportional to $1 / \sqrt{n}$, and therefore the time to reach the marked state from the superposition state is proportional to $\sqrt{n}$. This gives optimal spatial search and explains how the optimum $\gamma$ for the system can be found.

Long-range interaction model.-_Long range interactions can be realized in one-dimensional ion-trap systems, where spin-spin couplings are generated through laser-induced forces that off-resonantly drive vibrational modes of the ion chain [21,22]. This interaction is well described by the $X Y$ model with Hamiltonian $[19,20]$

$$
H=\sum_{i<j} J_{i j}\left(\sigma_{i}^{x} \sigma_{j}^{x}+\sigma_{i}^{y} \sigma_{j}^{y}\right),
$$

where the interaction strength, $J_{i j}$, is dependent on the distance between the spins,

$$
J_{i j}=\frac{1}{|j-i|^{\alpha}}+\frac{1}{|n-(j-i)|^{\alpha}},
$$

considering a closed one-dimensional spin chain.

In order to demonstrate optimal spatial search for this model, we must show that the time to reach the maximum fidelity scales $\sim \sqrt{n}$. Figure 1 (a) compares numerically simulated time to reach the maximum fidelity with an analytical prediction $\pi / 2 \sqrt{n / F_{\infty}(\alpha)}$, where we have analytically found an approximation of the fidelity for large $n$, $F_{\infty}(\alpha)$ (see the Supplemental Material [34]). We observe that the search time from numerical simulations closely follows the analytical times for $\alpha<1.5$. This implies spatial search in optimal $\mathcal{O}(\sqrt{n})$ time. Furthermore, Fig. 1(b) shows that the fidelity for $\alpha<1.5$ is closely approximated by $F_{\infty}(\alpha)$. The asymptotic value of $F_{\infty}(\alpha)$ is displayed in the inset of Fig. 1(a). Thus, whenever $F_{\infty}(\alpha)$ is asymptotically nonzero, there is optimal spatial search 
(a)

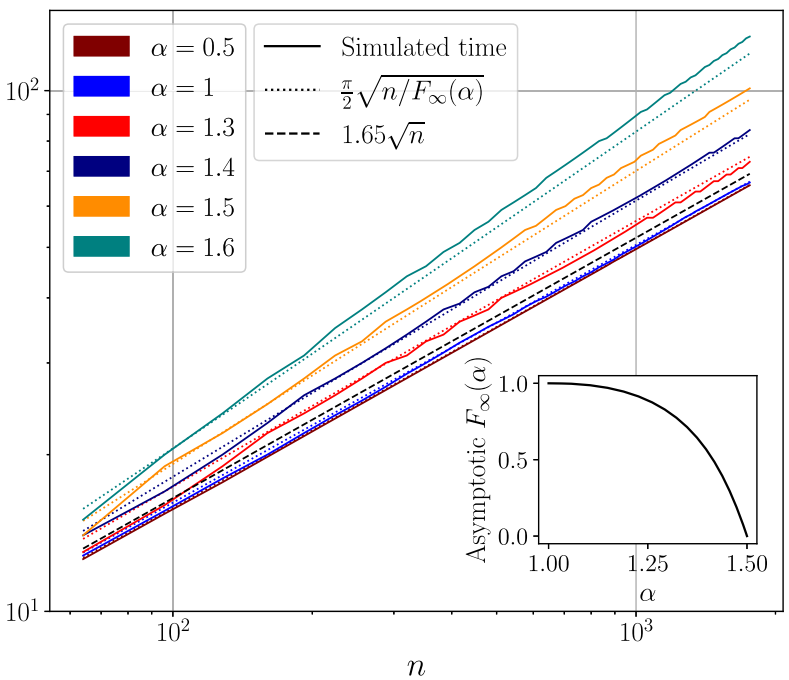

(b)

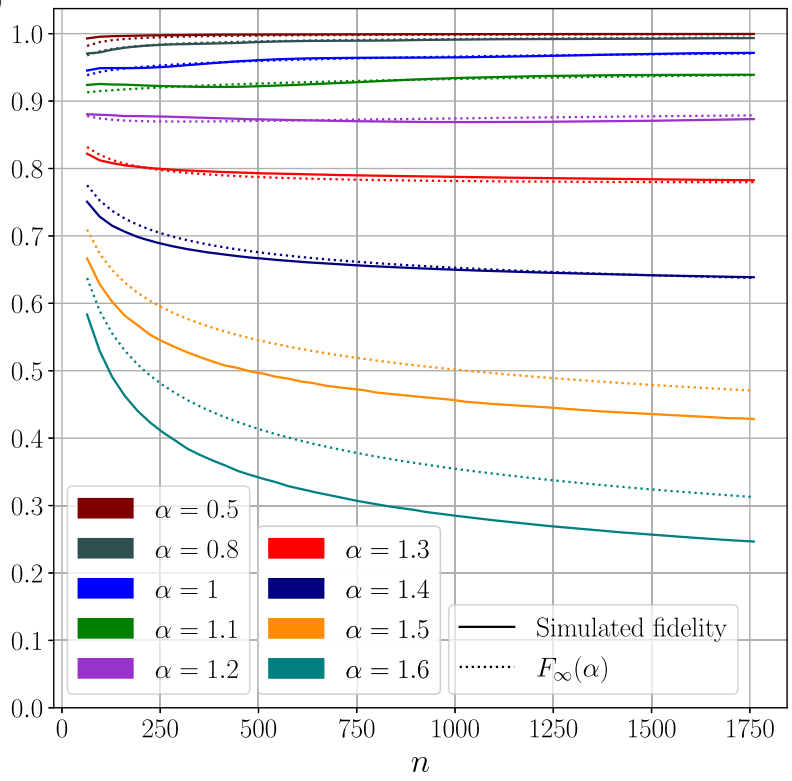

FIG. 1. (a) Time to reach the maximum fidelity of the marked state against the number of spins $n$. (b) Maximum fidelity of the spatial search against $n$. Numerical results are compared with the analytical predictions: $\pi / 2 \sqrt{n / F_{\infty}(\alpha)}$ for time, and $F_{\infty}(\alpha)$ for the fidelity, as defined in Eq. (3). The line (dashed black) $1.65 \sqrt{n}$ is plotted in (a) as a reference for how well the time scales as $\mathcal{O}(\sqrt{n})$. Inset: asymptotic value of $F_{\infty}(\alpha)$, in the limit $n \rightarrow \infty$, as a function of $\alpha$ using the approximation of $F_{\infty}(\alpha)$ from Eq. (9). (Supplemental Material contains an additional plot for time with fit parameters and a plot for fidelity that shows the asymptotic limits.)

in $\mathcal{O}(\sqrt{n})$ time. We also note that for $\alpha \leq 1.1$, the fidelity is high (above 0.9) for low $n$ and approaches its asymptotic value relatively quickly. Even before the asymptotic value is reached, the scaling with $n$ is not significant and essentially gives optimal spatial search for all $n$. We have confirmed that these results are irrespective of the marked state chosen. In particular, the fidelity is high for $\alpha=1$ and reaches over 0.97 for 1760 spins, while for $\alpha=1.4$ the fidelity is low, around 0.6 for $n \sim 3 \times 10^{6}$ (not shown).

Optimal search regime.-We apply the criterion for the optimality of quantum search from Ref. [11], to investigate the $\alpha$ values that permit optimal spatial search. This criterion is valid when the spectral condition

$$
\Delta \geq c n^{-1 / 2}
$$

is satisfied, where $c$ is a small positive constant, and $\Delta(\alpha)=1-\tilde{\lambda}_{n-1}(\alpha)$ is a rescaled spectral gap $-\tilde{\lambda}_{k}$ are the eigenvalues of $H$ in increasing order, rescaled such that the largest eigenvalue is 1 and the smallest is 0 . When the spectral condition is satisfied, for large $n$, the fidelity $F_{\infty}$ and time $T$ are related by $T \approx(\pi / 2) \sqrt{n / F_{\infty}}$. Therefore, if $F_{\infty}(\alpha) \rightarrow 0$ asymptotically, optimal spatial search in $\mathcal{O}(\sqrt{n})$ time is not possible.

The region where the spectral condition applies, as determined by Eq. (7), is dependent on $\alpha$. Asymptotically with respect to $n$, we find the spectral gap for $\alpha<3$ and $\alpha \neq 1$,

$$
\Delta(\alpha) \sim 1-\frac{1-\frac{g_{0}(\alpha)}{f(\alpha)} n^{1-\alpha}}{1-\frac{2}{f(\alpha)} \frac{n^{1-\alpha}}{\alpha-1}},
$$

where $f(\alpha)=4 \zeta(\alpha)-2^{2-\alpha} \zeta(\alpha), g_{0}(\alpha)=-2^{\alpha} \pi^{\alpha-1} \sin (\alpha \pi / 2) \times$ $\Gamma(1-\alpha), \zeta(\alpha)$ is the Riemann zeta function, and $\Gamma(1-\alpha)$ is the gamma function. This is proved in the Supplemental Material. For $\alpha<1$, we therefore find $\Delta(\alpha)=\mathcal{O}(1)$, which satisfies the spectral condition. Using Lemma 5 from Ref. [11], $\Delta(\alpha)(1-1 / n) \leq F_{\infty}(\alpha) \leq 1$. Therefore, $F_{\infty}(\alpha)$ must tend to a constant. This proves optimal spatial search exists for $\alpha<1$. Numerically this fidelity tends to 1 , see Fig. 1(b).

For $\alpha>1.5$, optimal spatial search cannot exist due to the Lieb-Robinson bounds for long-range interactions [38-40]. These bounds give an effective light cone for the maximum correlation distance $r$ after time $t$. In a one-dimensional free-particle system $t=\mathcal{O}\left(r^{\alpha-1}\right)$ for $1<\alpha<2$, while $t=\mathcal{O}(r)$ for $\alpha \geq 2$ [41]. For spatial search, since the maximum distance is $r=\mathcal{O}(n)$, we find that the time must be lower bounded by $t=\mathcal{O}\left(n^{\alpha-1}\right)$, thus showing that optimal spatial search with $t=\mathcal{O}(\sqrt{n})$ is not possible for $\alpha>1.5$.

For $1<\alpha<1.5$, we find $\Delta(\alpha)=\mathcal{O}\left(n^{1-\alpha}\right)$. Combining this scaling with Eq. (7), we have therefore proved that the spectral condition is asymptotically satisfied for $\alpha<1.5$. This is demonstrated numerically in Fig. 2. Moreover, in the Supplemental Material, we find the asymptotic expansion

$$
F_{\infty}(\alpha) \approx \frac{2\left(n^{\alpha-2} \zeta(\alpha-1)+\frac{2^{\alpha-2}}{2-\alpha}\right)^{2}}{n^{2 \alpha-3} \zeta(2 \alpha-2)+\frac{2^{2 \alpha-3}}{3-2 \alpha}},
$$




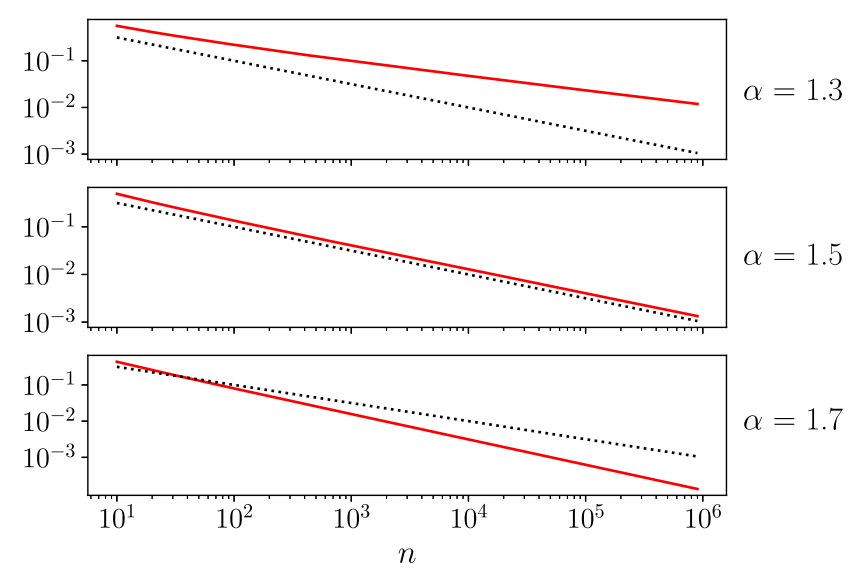

FIG. 2. The spectral condition is shown by comparing $\Delta(\alpha)$ from Eq. (8) (solid red line) with $n^{-1 / 2}$ (dotted black line) for various $\alpha$. The small positive constant $c$ from Eq. (7) has been ignored, as it provides a constant shift.

and prove that $F_{\infty}(\alpha)$ also approaches a nonzero value for $1<\alpha<1.5$. Therefore, optimal spatial search exists for $\alpha<1.5$, although for $\alpha$ close to 1.5 the fidelity converges to a low value, despite being optimal. The inset of Fig. 1(a) illustrates the continuous transition between the two regimes where, asymptotically, optimal spatial search exists $(\alpha<1.5)$ or is impossible $(\alpha>1.5)$. This curve is reminiscent of the behavior of the order parameter when the controls pass a phase transition point, although with a different physical explanation. The asymptotic fidelity $F_{\infty}(\alpha)$ predicts perfect search for $\alpha=1$, with a significant decrease after $\alpha=1.3$, before reaching 0 at $\alpha=1.5$, see the Supplemental Material for details.

Dephasing noise.-In a physical implementation, the dephasing of the qubits is the principal impediment. A pure dephasing which allows coherence to be lost without energy exchange with the environment, is the most dominant in ion traps, for example [42]. This can be modeled as random local field fluctuations by adding a noise term to the diagonal elements of the system Hamiltonian. The noise has a mean of 0 and is sampled from a Gaussian distribution, the standard deviation of which defines the noise parameter. Our results were obtained by generating 100 such Hamiltonians, running the spatial search for each one, and averaging over the outputs.

Figure 3 shows the evolution of the quantum walk on a closed spin chain of 256 spins with $\alpha=1$ at four different levels of noise. We find that the quantum walk on the ring is reasonably robust against dephasing and the maximum fidelity significantly falls when the noise level is greater than 0.02 . This corresponds to the field fluctuations being on the order of $2 \%$ of the field used to differentiate the marked state. The time to reach the maximum fidelity is also only minimally decreased at this noise level. The robustness of a quantum walk on a spin chain to small fluctuations in the interactions between sites has been

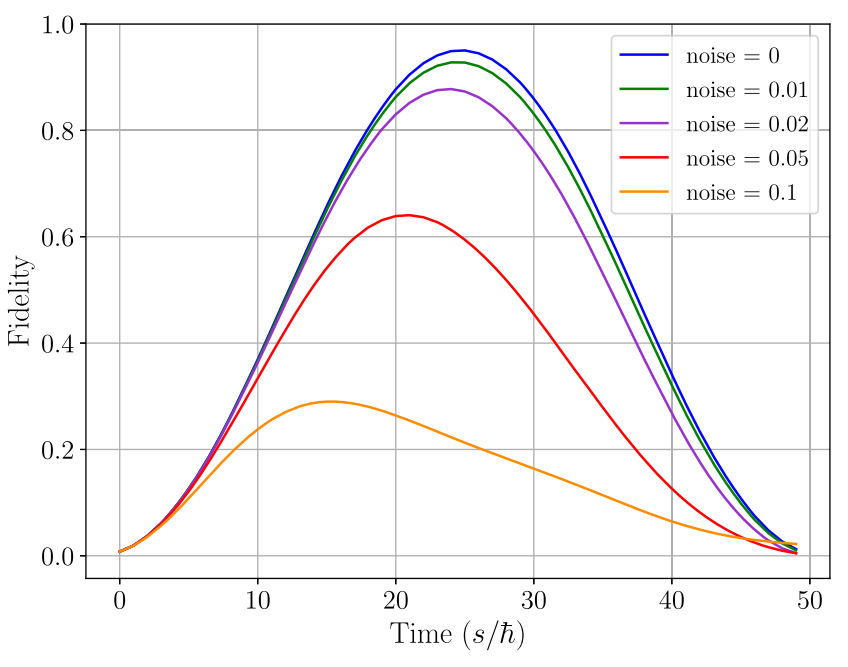

FIG. 3. Fidelity of the marked state over time for a closed spin chain of 256 spins with $\alpha=1$. Noise is the standard deviation of a Gaussian distribution for the local field applied to each site. The noise can be compared to the marked site field which has a magnitude of 1 .

shown analytically in the Supplemental Material of Ref. [43].

Discussion.-We have shown the possibility of a quantum speedup in a NISQ device with permanent long-range interactions. It can also be regarded as a quantum computation application of a quantum simulator-in this sense, it is even less demanding than what is envisaged in a typical NISQ device because it does not require individual gates between distinct qubits, it merely requires a timeindependent many-body Hamiltonian to be switched on, and then switched off after a specified interval of time. Our result is that optimal spatial search is physically realizable in one-dimensional spin chains through long-range interactions that decay as a $1 / r^{\alpha}$ potential. We have demonstrated analytically and confirmed numerically that optimal spatial search in $\mathcal{O}(\sqrt{n})$ time exists for $\alpha<1.5$. As $\alpha$ approaches 1.5 , however, the fidelity becomes impractically low. For experimentally realistic $n$, around 50 to 100 , the interaction range $\alpha \lesssim 1.2$ gives a fidelity above 0.88 . We have also shown that dephasing noise of $\sim 1 \%$ of the marking field only slightly reduces fidelity. Therefore, without considering specific implementations, we argue that optimal spatial search could be achieved experimentally for $\alpha \lesssim 1$.2. Long-range interactions with these values of $\alpha$ have been demonstrated in ion traps $[20,21,44,45]$. Using key results from Chakraborty et al. [11], we were able to show that an interaction strength of $\alpha=1.5$ defines a phase transitionlike point for optimal spatial search, where the asymptotic fidelity behaves like an order parameter.

Although we have shown optimal spatial search for the ring geometry of a closed one-dimensional chain, optimal scaling also exists for open spin chains, with the time to reach marked states at the edge of the chain being longer 
than central marked states. We can motivate the investigation of the ring as equivalent to the central spins of a much longer open chain. We also note that optimal spatial search should exist for the more connected twodimensional periodic lattice with long-range interactions, likely with a transition at a higher value of $\alpha$.

From a physical point of view, the spatial search algorithm allows detection of a local magnetic field faster than is possible classically. It could therefore find use in quantum sensing as a protocol for locating short-lived local magnetic fields along a spin chain-or extended to a twodimensional lattice. Perhaps such a device could be used for recognizing features in image processing faster than classically possible if the image is encoded as an array of fields that mark different qubits. By comparison with the theoretical maximum for the fidelity, the spatial search algorithm could also be used to establish how well coupled the long-range interactions of a spin chain are.

D. L., A. B., and N.F. acknowledge support from the EPSRC Centre for Doctoral Training in Delivering Quantum Technologies, Grant Ref. EP/S021582/1. L. B. acknowledges support by the program "Rita Levi Montalcini” for young researchers.

*dylan.lewis.19@ucl.ac.uk

[1] L. K. Grover, in Proceedings of the Annual ACM Symposium on Theory of Computing, Vol. Part F129452 (Association for Computing Machinery, New York, New York, USA, 1996), pp. 212-219.

[2] C. H. Bennett, E. Bernstein, G. Brassard, and U. Vazirani, SIAM J. Comput. 26, 1510 (1997).

[3] A. M. Childs and J. Goldstone, Phys. Rev. A 70, 022314 (2004).

[4] L. Novo, S. Chakraborty, M. Mohseni, H. Neven, and Y. Omar, Sci. Rep. 2015 5:1 5, 13304 (2015).

[5] S. Chakraborty, L. Novo, A. Ambainis, and Y. Omar, Phys. Rev. Lett. 116, 100501 (2016).

[6] S. Chakraborty, L. Novo, S. Di Giorgio, and Y. Omar, Phys. Rev. Lett. 119, 220503 (2017).

[7] L. Novo, S. Chakraborty, M. Mohseni, and Y. Omar, Phys. Rev. A 98, 022316 (2018).

[8] T. G. Wong, K. Wünscher, J. Lockhart, and S. Severini, Phys. Rev. A 98, 012338 (2018).

[9] T. Osada, B. Coutinho, Y. Omar, K. Sanaka, W. J. Munro, and K. Nemoto, Phys. Rev. A 101, 022310 (2020).

[10] R. Sato, T. Nikuni, and S. Watabe, Phys. Rev. A 101, 022312 (2020).

[11] S. Chakraborty, L. Novo, and J. Roland, Phys. Rev. A 102, 032214 (2020).

[12] J. Kempe, Quantum Random Walks: An Introductory Overview (2003).

[13] E. Farhi and S. Gutmann, Quantum computation and decision trees, Technical Report, 1998.

[14] A. M. Childs, R. Cleve, E. Deotto, E. Farhi, S. Gutmann, and D. A. Spielman, in Proceedings of the Thirty-Fifth ACM
Symposium on Theory of Computing-STOC '03 (ACM

Press, New York, New York, USA, 2003), p. 59.

[15] J. Preskill, Quantum 2, 79 (2018).

[16] I. M. Georgescu, S. Ashhab, and F. Nori, Rev. Mod. Phys. 86, 153 (2014).

[17] T. Osada, K. Sanaka, W. J. Munro, and K. Nemoto, Phys. Rev. A 97, 062319 (2018).

[18] A. M. Childs and Y. Ge, Phys. Rev. A 89, 052337 (2014).

[19] K. Kim, M. S. Chang, R. Islam, S. Korenblit, L. M. Duan, and C. Monroe, Phys. Rev. Lett. 103, 120502 (2009).

[20] B. P. Lanyon, C. Maier, M. Holzäpfel, T. Baumgratz, C. Hempel, P. Jurcevic, I. Dhand, A. S. Buyskikh, A. J. Daley, M. Cramer, M. B. Plenio, R. Blatt, and C. F. Roos, Nat. Phys. 13, 1158 (2017).

[21] P. Richerme, Z. X. Gong, A. Lee, C. Senko, J. Smith, M. Foss-Feig, S. Michalakis, A. V. Gorshkov, and C. Monroe, Nature (London) 511, 198 (2014).

[22] N. Friis, O. Marty, C. Maier, C. Hempel, M. Holzäpfel, P. Jurcevic, M. B. Plenio, M. Huber, C. Roos, R. Blatt, and B. Lanyon, Phys. Rev. X 8, 021012 (2018).

[23] A. Micheli, G. K. Brennen, and P. Zoller, Nat. Phys. 2, 341 (2006).

[24] B. Yan, S. A. Moses, B. Gadway, J. P. Covey, K. R. Hazzard, A. M. Rey, D. S. Jin, and J. Ye, Nature (London) 501, 521 (2013).

[25] A. Browaeys and T. Lahaye, Nat. Phys. 16, 132 (2020).

[26] C. F. Chen and A. Lucas, Phys. Rev. Lett. 123, 250605 (2019).

[27] J. Zhang, G. Pagano, P. W. Hess, A. Kyprianidis, P. Becker, H. Kaplan, A. V. Gorshkov, Z. X. Gong, and C. Monroe, Nature (London) 551, 601 (2017).

[28] Z. Eldredge, Z. X. Gong, J. T. Young, A. H. Moosavian, M. Foss-Feig, and A. V. Gorshkov, Phys. Rev. Lett. 119, 170503 (2017).

[29] M. C. Tran, A. Deshpande, A. Y. Guo, A. Lucas, and A. V. Gorshkov, arXiv:2010.02930.

[30] E. Farhi and S. Gutmann, Phys. Rev. A 57, 2403 (1998).

[31] M. F. Maghrebi, Z.-X. Gong, and A. V. Gorshkov, Phys. Rev. Lett. 119, 023001 (2017).

[32] T. Byrnes, G. Forster, and L. Tessler, Phys. Rev. Lett. 120, 060501 (2018).

[33] C. Cafaro and P. M. Alsing, Int. J. Quantum. Inform. 17, 1950025 (2019).

[34] See Supplemental Material at http://link.aps.org/supplemental/ 10.1103/PhysRevLett.126.240502 with this Letter for detailed analysis, which includes Refs. [35-37].

[35] NIST Digital Library of Mathematical Functions, https:// dlmf.nist.gov/ Release 1.1.0 of 2020-12-15 (2020).

[36] G. Nemes, Proc. R. Soc. A 473, 20170363 (2017).

[37] Y. Lee, J. Number Theory 134, 38 (2014).

[38] E. H. Lieb and D. W. Robinson, Commun. Math. Phys. 28, 251 (1972).

[39] M. C. Tran, A. Y. Guo, Y. Su, J. R. Garrison, Z. Eldredge, M. Foss-Feig, A. M. Childs, and A. V. Gorshkov, Phys. Rev. X 9, 031006 (2019).

[40] T. Kuwahara and K. Saito, Phys. Rev. X 10, 031010 (2020). 
[41] M. C. Tran, C.-F. Chen, A. Ehrenberg, A. Y. Guo, A. Deshpande, Y. Hong, Z.-X. Gong, A. V. Gorshkov, and A. Lucas, Phys. Rev. X 10, 031009 (2020).

[42] C. Piltz, T. Sriarunothai, S. S. Ivanov, S. Wölk, and C. Wunderlich, Sci. Adv. 2, e1600093 (2016).

[43] I. Pitsios, L. Banchi, A. S. Rab, M. Bentivegna, D. Caprara, A. Crespi, N. Spagnolo, S. Bose, P. Mataloni, R. Osellame, and F. Sciarrino, Nat. Commun. 8, 1569 (2017).
[44] N. Friis, O. Marty, C. Maier, C. Hempel, M. Holzäpfel, P. Jurcevic, M. B. Plenio, M. Huber, C. Roos, R. Blatt, and B. Lanyon, Phys. Rev. X 8, 021012 (2018).

[45] G. Pagano, A. Bapat, P. Becker, K. S. Collins, A. De, P. W. Hess, H. B. Kaplan, A. Kyprianidis, W. L. Tan, C. Baldwin, L. T. Brady, A. Deshpande, F. Liu, S. Jordan, A. V. Gorshkov, and C. Monroe, Proc. Natl. Acad. Sci. U.S.A. 117, 25396 (2020). 